\title{
Optimization study of diamond-shaped inverted nine spot patterns in low permeability reservoirs of horizontal well
}

\author{
Chunsen Zhao, Bu Fan, Yatao Lu, Zhong Ting ,Tao Bo, Didi Wu \\ ${ }^{1}$ Department of Petroleum Engineering, Northeast Petroleum University, Daqing, Heilongjiang \\ 163318, China \\ ${ }^{2}$ Department of Petroleum Engineering, Northeast Petroleum University, Daqing, Heilongjiang \\ 163318, China \\ ${ }^{3}$ Sixth Oil Production Plant of Daqing Oilfield and Northeast Petroleum University, Daqing, \\ Heilongjiang 163114, China \\ a zhaochs@163.com, ${ }^{\mathrm{b}}$ zhongting505@126.com, ${ }^{\mathrm{c}}$ zhongting.55@163.com
}

Keywords: low permeability reservoir; horizontal well; shape factor; optimization.

\begin{abstract}
In recent years, many people have made some research in horizontal wells of low permeability oil reservoirs. In order to further refinement of the existing research, this paper utilized conformal transformation and Potential superposition principle method to optimize and analyze the productivity of rhombus inverted nine-spot horizontal well pattern in low permeability reservoirs. On the basis of other parameters are given, by analyzing the relationships of dimensionless length and shape factor, dimensionless length and dimensionless output of horizontal well, he optimal shape factor and optimal dimensionless output under different well net area, reservoir thickness, and dimensionless length in low permeability reservoirs was got. The results show that it is suitable for using little well net area in low permeability reservoirs.
\end{abstract}

\section{Introduction}

The study aim to flow potential study and optimal analysis of the horizontal rhombus inverted nine-spot well patterns so that can provide meaningful suggestions for the production of horizontal wells in low permeability reservoir. In consideration of the actual conditions and seepage characteristics of low permeability reservoir, unit exploitation of horizontal well, branch horizontal wells and vertical wells will have more intuitive effect in low permeability reservoirs. Applicative effectiveness of horizontal well technology in oilfield development are better than vertical well, especially for reserves employing of the low permeability reservoir.

\section{The general situation}

The employed, explored and explored untapped oil reserves in the domestic land, reserve volume of low permeability reservoir are separately $11 \%, 60 \%$ and over $50 \%{ }^{[1]}$. Low permeability reservoir are rich in resources and widely distributed, with characteristics of poor reservoir property, low permeability, low porosity, high primary water saturation, serious heterogeneity and relatively developmental natural fracture ${ }^{[2-7]}$. In consideration of the actual conditions and seepage characteristics of low permeability reservoir, unit exploitation of horizontal well, branch horizontal wells and vertical wells will have more intuitive effect in low permeability reservoirs.

\section{The well location analysis}

Suppose anti- nine spot fracturing wells have $\mathrm{m}$ row and $\mathrm{n}$ column, and well spacing is $2 \mathrm{~b}$, and well line is 2 . The ratio of the number of injection wells and production wells is $1: 3$, therefore $Q_{\text {water }}=3 \mathrm{Q}_{\text {oi1 }}$, and we can be set $Q_{\text {water }}=3 \mathrm{Q}_{\text {oil }}$, then $\mathrm{Q}_{\text {water }}=3 \mathrm{Q}$. According to 
the characteristics of anti- nine rhombus pattern , each well location coordinates as: +Q: (4na,4mb);((2n+1)a,(2m+1)b);((4n-2)a,(4m-2)b); -3Q: ((4n-2)a,4mb);(4na,(4m-2)b).

By using the conformal transformation and the superposition theory, deduced the potential of fracturing wells at any point of any space in formation[8].

$$
\Phi(x, y)=\frac{Q}{2 \pi h} \operatorname{arch}\left[\frac{1}{\sqrt{2} \sin \frac{\pi L}{2 a}}\left(\sin ^{2} \frac{\pi L}{2 a}+\operatorname{ch}^{2} \frac{\pi}{2 a} y-\cos ^{2} \frac{\pi}{2 a} x+\sqrt{\left(\sin ^{2} \frac{\pi L}{2 a}+\operatorname{ch}^{2} \frac{\pi}{2 a} y-\cos ^{2} \frac{\pi}{2 a} x\right)^{2}-4 \sin ^{2} \frac{\pi}{2 a} x \operatorname{ch}^{2} \frac{\pi}{2 a} y \sin ^{2} \frac{\pi L}{2 a}}\right)^{\frac{1}{2}}\right]+c
$$

Annotation: $\Phi$---the force potential that Infinite row of producer caused; Q---the yield of producer, $\mathrm{m} 3 / \mathrm{t}$; h---reservoir thickness, $\mathrm{m}$; a---half of the pattern well spacing, $\mathrm{m}$; b--- half of the pattern row spacing, m; L--half of the length of horizontal well,m; c---this is a constant.

Because of the presence of ${ }^{[9]}$ starting pressure gradient, fluid percolation characteristics of low permeability reservoir doesn't strictly obey the Darcy's law and the corresponding kinematic equations. Therefore, we should consider the influence of starting pressure gradient in the theoretical calculation of low permeability reservoirs, and the flow quantity of the XY plane in low permeability reservoir can be expressed as:

$$
Q=\frac{\pi K h\left(\Delta p-G \sqrt{a^{2}+b^{2}}\right)}{\mu A}
$$

Annotation: G--- staring pressure gradient of low permeability reservoir, $\mathrm{Pa} ; \Delta \mathrm{p}$---pressure difference, Pa; $\mu$---viscosity, $\mathrm{mPa} \cdot \mathrm{s}$; K--- permeability, $10^{-3} \mu \mathrm{m}^{2}$.

In order to facilitate the analysis, the dimensionless rate of the horizontal well are defined as:

$$
Q_{\mathrm{D}}=\frac{Q \mu}{\pi K h \Delta p}
$$

The dimensionless potential difference of the XY plane are:

$$
R_{\mathrm{o}}=\frac{1}{Q_{\mathrm{D}}}
$$

Considering the filtrational resistance of horizontal well and anisotropy of reservoir in the YZ plane, the dimensionless potential difference can be expressed as:

$$
R_{\mathrm{i}}=\frac{h}{2 l} \ln \frac{h}{2 \pi r_{\mathrm{w}}}
$$

Therefore, the accurate solutions of dimensionless rate of diamond-shaped inverted nine-spot horizontal well patterns in low permeability can be expressed as:

$$
Q_{D}=\frac{1}{2 R_{i}+R_{0}}
$$

\section{Optimization analysis of diamond-shaped inverted nine spot patterns of horizontal well}

The pattern shape factor and the dimensionless length of horizontal well can affect dimensionless rate of the horizontal wells in low permeability reservoir. If the given pattern areas were unchanged and the length of horizontal well were also in certain circumstances, its yield are different with changing the pattern shape. Therefore, there must be an optimal well pattern shape unit that make the maximum yield of horizontal well , under the same conditions of the pattern area and the length of horizontal well . Consequently, the study try to find the relationship between dimensional length and well optimum shape factor by using Eq. 3, so as to realize the optimization of well pattern.

\section{(1) Optimization analysis of well patterns}

Specific practices of well pattern optimization are that calculate the optimal well pattern shape factor() when dimensionless yield of horizontal well are maximum, by using Eq. 3 and Mathematic mathematical software, under the condition that give the other parameters and make the dimensionless length of fracturing well unchanged. Therefore, we can obtain the relation curve 
between the optimal well pattern shape factor and dimensionless length of horizontal well , as well as between dimensionless rate of horizontal well and corresponding dimensionless length. In this pattern area and reservoir thickness conditions, we should record dimensionless length Fop (ID), corresponding dimensionless yield(QD) and Fop. In the study, we analysis the optimization process of the yield in XY plane.

\section{(2)The optimized results}

In optimized calculation, taking into account the specific situation of oilfield, we can set up the various parameters that pressure is 3MPa, the pattern area are separately $10000 \mathrm{~m} 2$ and $40000 \mathrm{~m} 2$ respectively, and reservoir thickness are respectively $10 \mathrm{~m}, 30 \mathrm{~m}, 50 \mathrm{~m}$. The results of the analysis were shown in fig. 1 to fig. 3

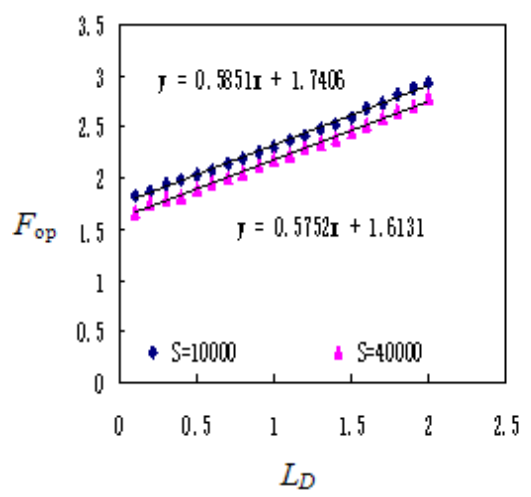

Fig.1 The relationship between $L_{D}$ and Fop when $\mathrm{S}$ are $10000 \mathrm{~m}^{2}, 40000 \mathrm{~m}^{2}$

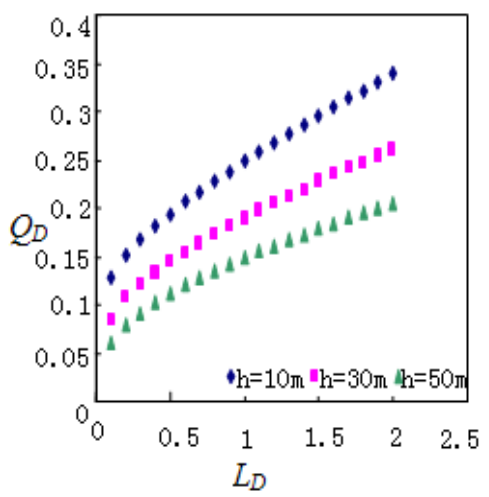

Fig.2 The relationship between $L_{D}$ and $Q_{D}$ when $\mathrm{S}$ are $10000 \mathrm{~m}^{2}$

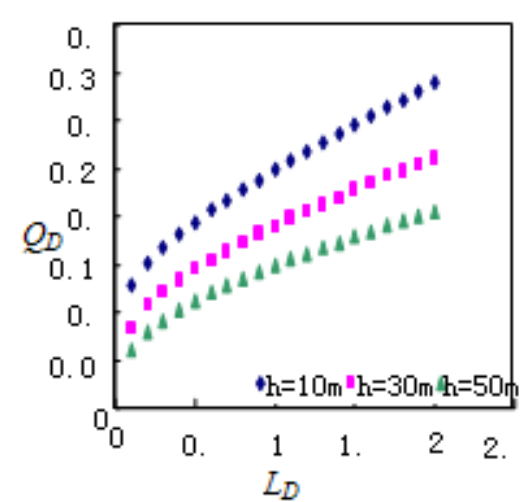

Fig.3 The relationship between $L_{D}$ and $Q_{D}$ when $\mathrm{S}$ are $40000 \mathrm{~m}^{2}$

By means of analysis data, there must be a well pattern shape factor to make the dimensionless yield of horizontal well maximum, and the shape factor is the optimal shape factor now when the pattern area and the dimensionless length of horizontal well are unchanged, in diamond-shaped inverted nine spot patterns of low permeability reservoirs.

According to Eq. 3 and fig.1, we can find that the optimal well pattern shape factor are constant ,and we can ignore the effect of reservoir thickness to well pattern shape factor when the pattern area of diamond-shaped inverted nine spot patterns are definite and the other parameters are unchanged. Moreover, the optimal shape factor will change with the dimensionless length changed, in the same condition of the pattern areas. Furthermore, the optimal well pattern shape factor will be smaller with the dimensionless length enlarged when dimensionless length are the same. And the relationship between dimensional length of horizontal well and the optimal well pattern shape factor conform to linear relationship.

Through the above figure, we can find that dimensionless rate will broaden with dimensionless length increasing. The effect of size of pattern area will be larger when reservoir thickness get larger. Besides the dimensionless rate of the small pattern area are greater than the dimensionless rate of the large pattern area, under the same condition. Consequently, it will get better production effect when we adopt small well spacing in low permeability reservoir.

\section{Summary}

Through research and analysis, we come to the following conclusions:

(1) According to the conformal transformation and overlay principle of force potential, we deduced productivity formula of horizontal well pattern of diamond-shaped inverted nine spot patterns and superposition using conformal change and proceed optimization analysis.

(2) The study discuss the relationship between the dimensionless length of horizontal wells and w the optimal well pattern shape factor, the optimal dimensionless yield. Moreover, we define explicitly the effect relationship between reservoir thickness and pattern area, and believe that that using small well spacing can get better production effect in low permeability reservoir. 


\section{References}

[1] Guangcai Tang, Weiying Wang, Huang Lei, et al. Effect factorsof horizontal well productivity in low permeable reservoir [J]. Tu Ha You Qi, 2009,14(3):231-233.

[2] Renpu Wan. Exploited technique of horizontal wells of different reservoir types in China [M]. Beijing: Petroleum Industry Press, 1997.

[3] Yingzhi Zhang, Tiejun Yang, et al. The development technology research of the extra low permeability reservoir [M]. Beijing: Petroleum Industry Press, 2004.

[4] Yunting $\mathrm{Xu}, \mathrm{Xu}$ Kai, Yonggui Guo, et al. Study and application on filtration mechanism of low permeability reservoir[M]. Beijing: Petroleum Industry Press, 2006.

[5] Huifen Xia, Qitai Mei, Fujun Li. Process and technology of exploitation in low permeability reservoir[M]. Beijing: Petroleum Industry Press, 1996.

[6] Xiaodong Wang. The research on unsteady seepage of horizontal well[D]. Hebei: The seepage flow mechanics of CHINESE ACAD SCI,1998.

[7] Carvatho, R.de S, et al. Transient Pressure Behavior for Horizontal Wells in Naturally

Fractured Reservoir. SPE 18302.

[8] Xiangyan Kong. Higher Seepage mechanics [M]. Hefei: The press of China Science \& Technology University, 1999.

[9] Van Domelen M S. Optimizing fracture acidizing treatment design by integrating core testing field testing and computer simulation[C]. SPE22393, 1992:24-27. 